\title{
INTRODUCING A NOVEL FAULT TOLERANT ROUTING PROTOCOL IN WIRELESS SENSOR NETWORKS USING FUZZY LOGIC
}

\author{
Mostafa Vakili Fard ${ }^{1}$, Sayyed Majid Mazinani ${ }^{2}$, Sayyed Ahmad Hoseini ${ }^{3}$ \\ ${ }^{1}$ Department of Computer Engineering, Science and Research IAU, Neyshabur, Iran \\ ${ }^{2}$ Department of Computer Engineering, Imam Reza University, Mashhad , Iran \\ ${ }^{3}$ Department of Computer Engineering, , payam Noor University, Mashhad , Iran
}

\begin{abstract}
Although routing is one of the key network issues, the challenges facing WSN makes the subject even more demanding. In this study, we propose a novel fault tolerant routing protocol in wireless sensor networks using fuzzy logic. We approach the problem by breaking it down into two parts. The first sub problem is designing a routing module and the second one retrofitting the designed module in a way that answering to these two sub problems leads to the final solution of the main problem. To deal with the first stage, by using fuzzy logic, we design a routing module; then for the second stage, the module enters fault tolerant routing in order that it be tolerant against routing faults. In this study, the term "network failure" is figuratively equal to "node failure".
\end{abstract}

\section{KEYWORDS}

wireless sensor networks, routing, fault tolerance, fuzzy logic.

\section{INTRODUCTION}

Routing in wireless sensor networks embraces a different concept comparing to other wireless communication networks due to a handful of fundamental characteristics; therefore, the researchers have faced new challenges. The very first challenge in wireless sensor networks is incapability of building a unique global address for each node. Consequently, the protocols, used in those wireless networks functioning under IP protocol, will be considered useless in such networks. The second challenge is that nearly all applications under WSN require transferring the sensed data from the surroundings to a certain unit, called the base station. As for the third challenge, the data traffic generated in these networks has a considerable redundancy. One event can be detected by different nodes and they accordingly send the same data. Such redundancies should be considered in order to use energy consumption in nodes and bandwidth more logically. The fourth challenges concern the limitations in data-saving energy, power computation, and node total capacity[1].

\section{REVIEW OF LITERATURE}

Fuzzy logic is used to recognize the values which are not one hundred percent true or false, and which are facing against classical logic that has been utilized in control systems thus, they are widely used in various systems, such as WSNs to name a few. Algorithm of [2] introduces a routing protocol which by using fuzzy logic obtains the node's residual energy, local density 
inside its sensed region, and time as input parameters of fuzzy module. This leads to a fair distribution in order to make a cluster. This protocol benefits from two fuzzy modules. Algorithm of [3] presents a routing protocol which selects the proper cluster head based on fuzzy logic. Selecting the proper cluster head has an impact on energy saving and extending the network lifetime. By using three parameters of energy, density and centricity this algorithm makes better decisions of fuzzy modules. In algorithm of [4], the head clusters are selected by the base station. Fuzzy controller is our best routing component. Its input parameters are the head cluster's center of gravity, node's remaining energy, and the network traffic. Algorithm of [5] presents a fault tolerant routing mechanism which uses a multi-path technique. Such algorithm uses separate paths in order to deliver the sensed data to the base station. Doing so, it selects a supporting head cluster for each head cluster. Algorithm of [6] presents a routing protocol with energy efficiency which uses the fixed delay and selects the next hop proactively utilizing both paths.

In this study, we propose a novel fault tolerant routing protocol in wireless sensor networks using fuzzy logic. Benefiting fault tolerant routing protocol paves the way of carrying on the routing operation in the presence of faults. Here, the term "network failure" is figuratively equal to "node failure".

\section{PROBLEM DEFINITION AND SOLVING}

We are breaking the problem down into two sub problems of designing a routing module based on fuzzy logic and of retrofitting it. We are hypothesizing flat network architecture and querybased network operation, along with a proactive routing and routing will happen at the time of generating the sensed data.

\subsection{Designing Routing Based On Fuzzy Logic}

The first hop to solve the early sub problem, the fuzzy module is designed based on the Mamdani fuzzy interference system. In Mamdani system, input and output are expressed in fuzzy. Figure 1 illustrates parts of a fuzzy system.

Fuzzy Inference System(FIS)

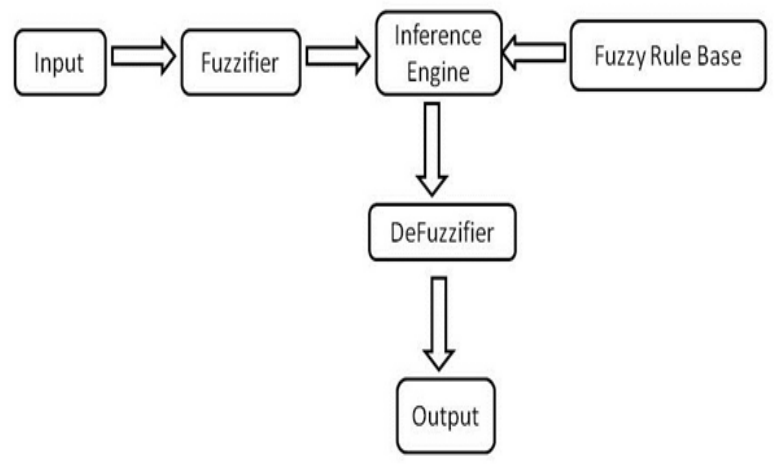

Fig 1. Fuzzy inference system components

Four parameters Residual energy of node, path traffic, numbers of hops to the base station, and packet priorities are input parameters of the fuzzy module. These parameters enter the fuzzy module through neighbour management unit (NMU). 
Figure 2 illustrates the function membership related to the node's residual energy. Table 1 illustrates the information concerning the other membership functions of fuzzy module.

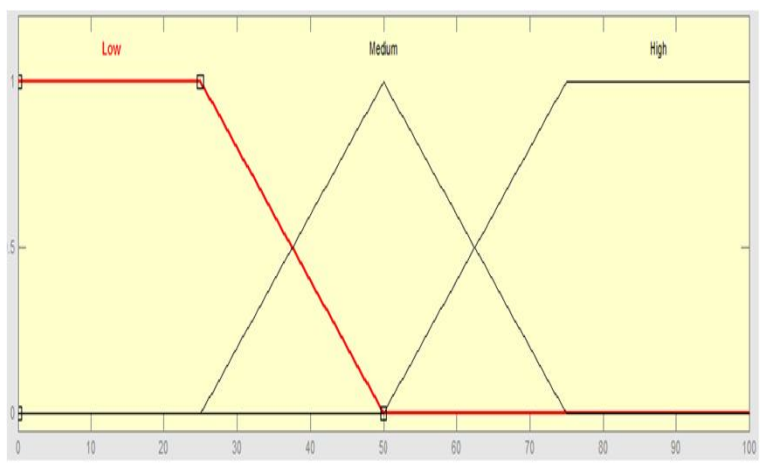

Fig 2. Residual energy membership function

According to figure 1, the fuzzy rules are used as one of fuzzy system parts. The following is the general idea of a fuzzy rule:

IF Variable Is Set Then Action

Since we have used the fuzzy module in routing designing, to receive satisfying output, we utilize center-of-gravity function in order to dismiss the output from its fuzzy mode. When a node contains sensed data, the NMU provides the requiring information for the fuzzy routing module. The output parameter is considered to be as the node goodness. Thus, the fuzzy inference engine calculates the node goodness value and singles out the most suitable node.

Table 1. Fuzzy module membership function

\begin{tabular}{|c|c|c|c|}
\hline & Low & Medium & High \\
\hline Path Traffic(\%) & $40<<0$ & $30<<80$ & $70<<100$ \\
\hline Number Of Hops & $0<<3$ & $2<<4$ & $3<<5$ \\
\hline Packet Priority & $0<<1$ & $0.5<<1.5$ & $1.5<<2$ \\
\hline Node Goodness & $0<<0.5$ & $0.25<<0.75$ & $0.6<<1$ \\
\hline
\end{tabular}

\subsection{The Fault Tolerating Step}

As mentioned earlier, the fault tolerating aims to let the routing operation happen in the presence of faults and, finally, the packets be delivered to the destination. On the other hand, redundancy is the very basis of retrofitting. So, we devise an alternate path besides the main path for each node's sensed data down to the destination. In this stage, the output taken from fuzzy routing designing is considered to be a black box. Of all the neighbour nodes, the one with maximum membership, selected as the next hop, places in the first best path and the node with the second ranking does in the second best path. The first best path is naming the main path and the second best one the alternate path. The NMU collects data relating to each node's membership in main and alternate paths. For a generating node, it's only possible to be a member of either the main or alternate path. Figure 3 illustrates our proposed routing mechanism. The yellow node is the node ready to send data; the main path is in green and the alternate path is in red arrows. 


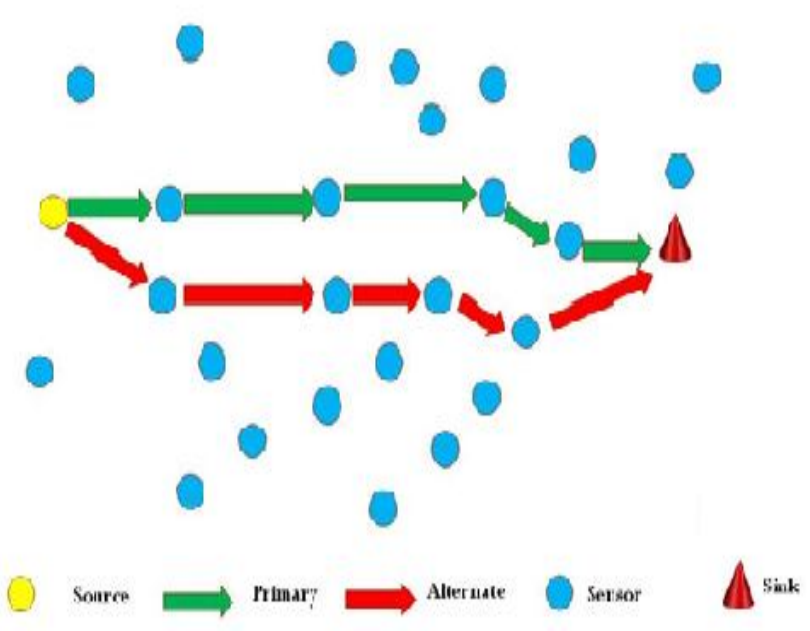

Fig 3. Proposed Routing Mechanism

\section{SIMULATING OPERATION}

As mentioned in fuzzy routing input, the priority of the packets is one of the key input parameters to the fuzzy module.

The packets are prioritized into three levels. Level Zero, the interested message distributing from the base station to the network; Level One, the packets distributing among the nodes and management neighbour units; Level Two, the sensed data by nodes. Levels with higher numbers establish superior priorities.

We assign three variants to the suggested protocol, and name Level 0, Level 1, and Level 3, as FTRF 0, FTRF 1, and FTRF 2 accordingly. In a way that the variant FTRF 0 assigns alternate paths for Levels 0,1 , and 2.

Having done the simulation in Castalia environment, we have compared three parameters of the network lifetime, consumed energy, and delivery ratio packets to the base station resulted from the mentioned variants. Figures 4, 5, and 6 illustrate the results taken from three variants. After studying the results, we pick the variant with the best result. Figure 4 illustrates scales of the sensed packets comparing to the residual-energy average. Gradually, with increasing of numbers of the sensed packets, and due to only one sort of redundant data, the variant FTRF 2 minimizes its consumption to a moderate level, in comparison to other two variants generating more redundant packets. Count ratio of sensed packets with percentage of critical nodes are used in order to calculate the network lifetime.

Figure 5 illustrates that with increasing of numbers of the sensed packets, the critical nodes in variant FTRF0 escalate, but the variant FRTF2 contains less critical nodes.

Figures 6 illustrates the comparison of parameters of the packet delivering in these three variants, where by increasing of sensed data generating nodes, packet delivering to the base station reduces -- a natural circumstance due to gradual consumption of nodes' energy. However, among the variants, FTRF2 benefits from a better delivery ratio than the others. 
International Journal of Computer Science \& Information Technology (IJCSIT) Vol 5, No 5, October 2013

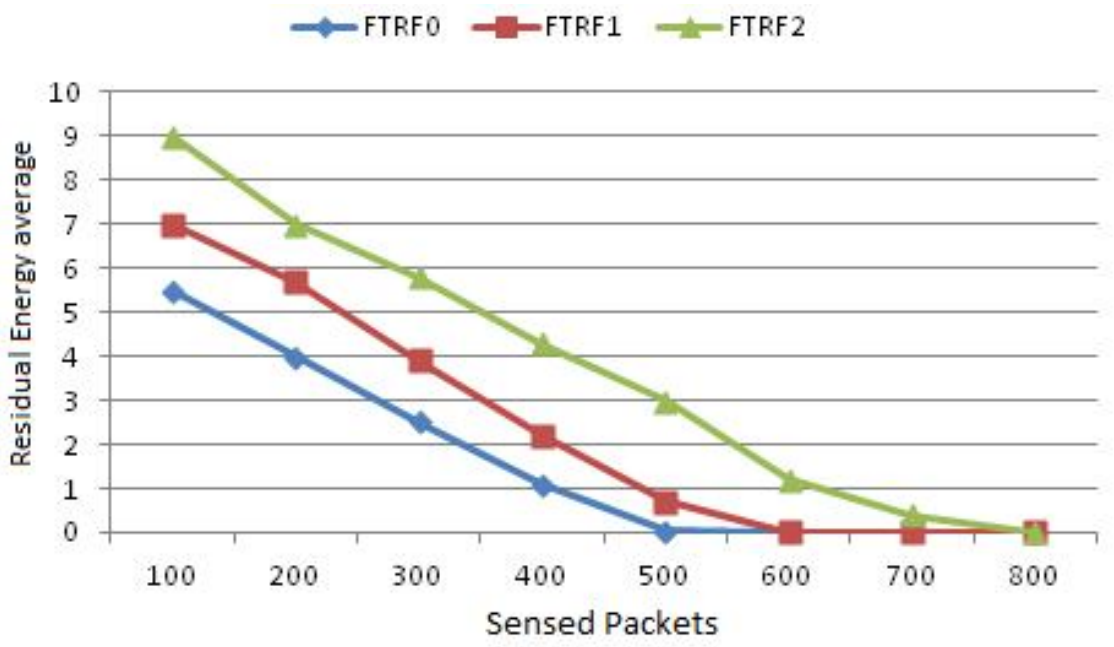

Fig 4. Residual energy ratio with sensed packets

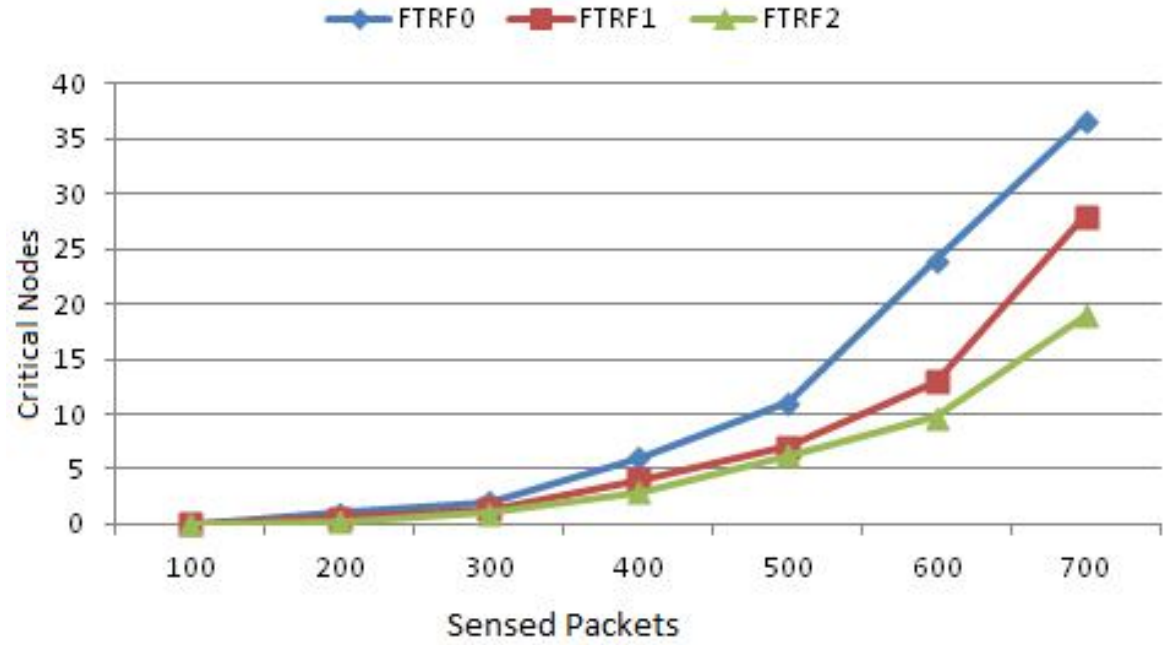

Fig 5. Critical nodes ratio with sensed packets 


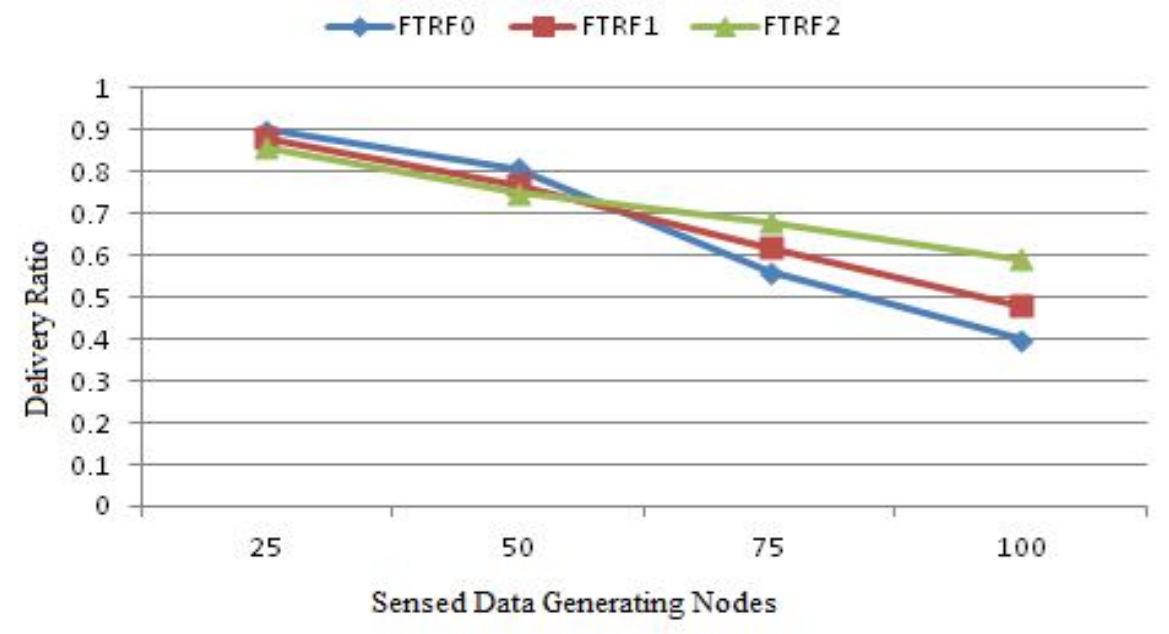

Fig 6. Delivery ratio with sensed data generating nodes

\section{CONCLUSION}

In this study, we propose a novel fault tolerant routing protocol in wireless sensor networks using fuzzy logic. After designing and routing based on fuzzy logic, we utilize multi-path redundancy and routing techniques in order to increase the suggested fault tolerant routing. Since, in this study, the term "network failure" is figuratively equal to "node failure", the fault tolerant routing, even facing node failures, must be capable of routing the packets to their final destination. The variant FTRF2 features longer lifetime and delivers more packets to their destination among the proposed redundancy of other variants.

In this study, there is no citation of recovering mechanism for routing failure. To ensure accuracy, moreover, the membership functions of fuzzy module input can increase in order to provide service for traffic requiring special service qualities.

\section{REFERENCES}

[1] K. Akkaya and M. Younes , "A survey on routing protocols for wireless sensor networks " , Ad Hoc Networks , vol. 3 , no. 3 , May 2005 , pp. 325 - 349

[2] Y.M Tashtoush and M.A Okour, Fuzzy Self-Clustering for WSN, IEEE/IFIP International Conference on Embedded and Ubiquitous Computing 2008.

[3] J.Kim, S.park, Y.Han and T.Chung, "CHEF:cluster head election mechanism using fuzzy logic in WSN", in proceeding of the ICACT , 2008,pp.654-65

[4] Joupe Anno ,Leonardo Barolli , “ A cluster head selection method for WSN base on fuzzy logic” , IEEE 2007 , pp. 337 - 348.

[5] D. Ganesan, R. Govindan, S. Shenker, and D. Estrin, "Highly-resilient, energy-efficient multipath routing in wireless sensor networks," vol. 5, pp. 11-25, Oct. 2001.

[6] Yuan, L.F.; Cheng, W.Q.; Du, X. An energy-efficient real-time routing protocol for sensor networks. Comp. Commun. 2007, 30, 2274-2283. 